\title{
Forewarned is forearmed: Benefits of remote ischemic preconditioning
}

\author{
Rupak Mukherjee, $\mathrm{PhD}$
}

\footnotetext{
From the Division of Cardiothoracic Surgery, Department of Surgery, Medical University of South Carolina, Charleston, SC; and the Ralph H. Johnson VA Medical Center, Charleston, SC.

Funding: Salary support from a National Institutes of Health grant (AG036954) and a SPiRE award from the Veteran's Administration (I21 RX001707).

Disclosures: Author has nothing to disclose with regard to commercial support.

Received for publication Oct 23, 2015; accepted for publication Oct 24, 2015; available ahead of print Nov 25, 2015 .

Address for reprints: Rupak Mukherjee, PhD, Division of Cardiothoracic Surgery, MSC 777, 114 Doughty St, Ste 338, Strom Thurmond Bldg, Charleston, SC 29426 (E-mail: mukherr@ musc.edu).

J Thorac Cardiovasc Surg 2016;151:786-7

$0022-5223 / \$ 36.00$

Copyright (c) 2016 by The American Association for Thoracic Surgery

http://dx.doi.org/10.1016/j.jtcvs.2015.10.089
}

Ischemic preconditioning (IPC) is a process in which a series of short bouts of ischemia that are followed by reperfusion confer to the affected organ protective effects from a subsequent prolonged ischemic insult. ${ }^{1}$ These beneficial effects of IPC on the ischemic myocardium were first described by Murry and colleagues ${ }^{2}$ in the mid-1980s, and subsequently confirmed by several laboratories. Moreover, these effects of IPC are not limited to the myocardium, but extend to organs such as the kidneys, brain, liver, and skin flaps, among others. Although the beneficial effects of IPC have been described, research continues to define cellular and molecular mechanisms. A recent search on PubMed, with the term "ischemic preconditioning," returned $>8900$ articles.

The clinical application of IPC, however, as a preemptive means to attenuate the effects of ischemia, is problematic. Specifically, IPC as originally conceived can be applied only in the setting of elective surgery, during which the organ that will be affected by ischemia during the procedure can be readily anticipated. This issue was addressed, at least partially, by the so-called phenomenon of remote ischemic preconditioning (RIPC).

Przyklenk and colleagues ${ }^{3}$ first demonstrated that the protective IPC stimulus could be applied to a different organ or tissue from the one that would be subjected to the ischemic insult. Specifically, they demonstrated that IPC in the circumflex artery region reduced the infarct size when the region perfused by the left anterior descending coronary artery was made ischemic. Studies since then have shown that performing IPC in organs other than the heart (such as the small intestine or kidney) reduced the extent of the infarct from occlusion of the coronary artery. ${ }^{4}$ This concept has now been expanded between noncardiac organs and tissues, such that it represents a general form of interorgan protection against ischemia-reperfusion injury. ${ }^{1}$

The study by Haapanen and colleagues ${ }^{5}$ from Dr Juvonen's group extends this principle to RIPC, to protect the spinal cord from ischemia-reperfusion injury by inducing

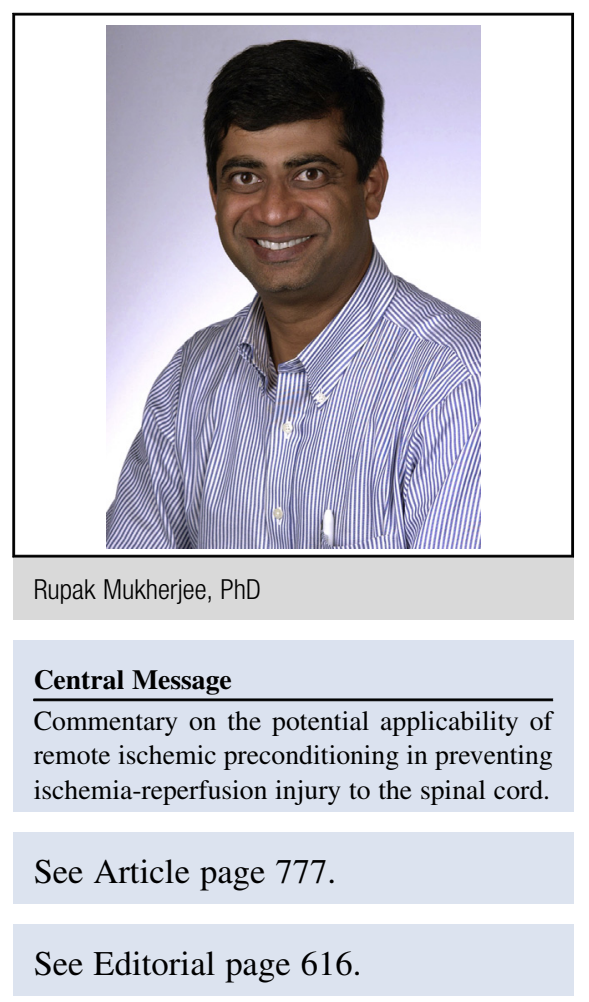

4 short bouts ( 5 minutes) of ischemia in 1 of the hind limbs. Specifically, RIPC was induced in pigs that were subjected to 4 bouts of hind-limb ischemia created by inflating a blood pressure cuff to $250 \mathrm{~mm} \mathrm{Hg}$ for 5 minutes, followed by reperfusion for 5 minutes. Pigs with similar instrumentation-but absent the 40 minutes of RIPC - were used in the control group for these studies.

Fifteen minutes after RIPC/sham, the investigators sequentially occluded the left subclavian artery and segmental arteries in the thorax. A $50 \%$ reduction of the amplitude of motor evoked potentials (MEPs) after stimulation of electrodes previously placed in the brain (on both sides of the sagittal suture) was used to determine that the spinal cord was made ischemic. The MEPs in the right hind limb of the animals in the RIPC group during the duration of sequential ischemia had significantly higher amplitude than those of animals that did not receive RIPC. This effect was attenuated in the left hind limb. Moreover, a brief neurologic assessment performed 24 hours after surgery on the animals' ability to stand and/or walk revealed a slightly better, but statistically nonsignificant, score in the RIPC group.

Although these results on the beneficial effects of hindlimb ischemia IPC on spinal cord function after ischemia are intriguing, limitations of this study must be recognized. 
First, a control cohort of pigs without RIPC and without spinal cord ischemia was not included. The inclusion of this group would have allowed for comparison of the effects of prolonged anesthesia on spinal cord function, postoperative neurologic assessment, as well as histology for the effects of ischemia on the spinal cord. This is particularly relevant because various anesthetic agents, including ketamine and sevoflurane (both used in this study), have been shown to confer at least some form of preconditioning effect in the setting of myocardial ischemia-reperfusion. ${ }^{6-10}$ However, whether the preconditioning effect of anesthetic agents extends to the spinal cord remains unknown. What is known - and this issue was carefully considered by the investigators-is that ketamine and fentanyl do not seem to affect MEPs. ${ }^{11,12}$

Second, the prolonged high pressure exerted by the cuff on the hind limb could have damaged the nerves and thereby limited clinical applicability. However, the authors showed that all pigs in the RIPC group were able to stand 24 hours postprocedure, which suggests that nerve damage, if any, was likely not significant. Third, the postprocedural mortality seemed to be higher in the group that received RIPC ( 3 of 10 pigs died) than in the control group ( 1 of 8 pigs died). Although this difference was unlikely to be statistically significant, given the small sample sizes used in this study, this finding does suggest that clinical implementation of RIPC must be carefully monitored, especially in the postoperative setting.

Finally, we did not attempt to determine molecular and cellular bases for the beneficial effects of RIPC. Despite this study shortcoming, the investigators were examining a clinically relevant process in a clinically relevant animal model. The use of a large animal model for these experiments is a strength of this study. Pigs have been shown to be an excellent model for simulation of several human forms of cardiovascular disease, and investigation of surgical techniques ${ }^{13}$; this study ${ }^{5}$ is no exception.

Protection of the spinal cord from ischemia and ischemia-reperfusion injury remains an important consideration during the performance of thoracoabdominal procedures, including aortic repair procedures. The study by Haapanen and colleagues ${ }^{5}$ provides proof-of-concept: Inducing IPC in a vascular bed remote from the site of surgery may confer benefits with respect to recovery from spinal cord ischemia encountered during elective surgery. Given that induction of RIPC as described here adds at least 40 minutes to the procedure, this technique may not be useful in cases of emergent surgery. Nevertheless, with enough forewarning (ie, an elective procedure), induction of RIPC has the potential to become a novel therapeutic modality to ameliorate the effects of ischemia (forearmed) on spinal cord injury in the surgical setting.

\section{References}

1. Hausenloy DJ, Yellon DM. Remote ischaemic preconditioning: underlying mechanisms and clinical application. Cardiovasc Res. 2008;79:377-86.

2. Murry CE, Jennings RB, Reimer KA. Preconditioning with ischemia: a delay of lethal cell injury in ischemic myocardium. Circulation. 1986;74 1124-36.

3. Przyklenk K, Bauer B, Ovize M, Kloner RA, Whittaker P. Regional ischemic 'preconditioning' protects remote virgin myocardium from subsequent sustained coronary occlusion. Circulation. 1993;87:893-9.

4. Gho BC, Schoemaker RG, van den Doel MA, Duncker DJ, Verdouw PD. Myocardial protection by brief ischemia in noncardiac tissue. Circulation. 1996;94:2193-200.

5. Haapanen HHJ, Arvola O, Anttila T, Starck T, Kalliio M, Anttila V, et al. Remote ischemic preconditioning protects the spinal cord after ischemic insult: an experimental porcine study. J Thorac Cardiovasc Surg. 2016;151:777-85.

6. Martini N, Preckel B, Thamer V, Schlack W. Can isoflurane mimic ischaemic preconditioning in isolated rat heart? Br J Anaesth. 2001;86:269-71.

7. Schlack W, Preckel B, Stunneck D, Thamer V. Effects of halothane, enflurane, isoflurane, sevoflurane and desflurane on myocardial reperfusion injury in the isolated rat heart. Br J Anaesth. 1998;81:913-9.

8. Obal D, Preckel B, Scharbatke H, Mullenheim J, Hoterkes F, Thamer V, et al. One MAC of sevoflurane provides protection against reperfusion injury in the rat heart in vivo. Br J Anaesth. 2001;87:905-11.

9. Molojavyi A, Preckel B, Comfere T, Mullenheim J, Thamer V, Schlack W. Effects of ketamine and its isomers on ischemic preconditioning in the isolated rat heart. Anesthesiology. 2001;94:623-9; discussion 5A-6A.

10. Belhomme D, Peynet J, Louzy M, Launay JM, Kitakaze M, Menasche P. Evidence for preconditioning by isoflurane in coronary artery bypass graft surgery. Circulation. 1999;100:II340-4.

11. Jacobs MJ, Meylaerts SA, de Haan P, de Mol BA, Kalkman CJ. Assessment of spinal cord ischemia by means of evoked potential monitoring during thoracoabdominal aortic surgery. Semin Vasc Surg. 2000;13:299-307.

12. Meylaerts SA, De Haan P, Kalkman CJ, Jaspers J, Vanicky I, Jacobs MJ. Prevention of paraplegia in pigs by selective segmental artery perfusion during aortic cross-clamping. J Vasc Surg. 2000;32:160-70.

13. Swindle MM, Makin A, Herron AJ, Clubb FJ Jr, Frazier KS. Swine as models in biomedical research and toxicology testing. Vet Pathol. 2012;49:344-56. 